\title{
Celiac crisis, atypical form of celiac disease onset in teenager - a case report
}

\author{
Daniela Ciobanu, Lorena Elena Melit, Ana-Maria Simon, \\ Nicoleta Tomsa, Cristina Oana Marginean \\ Pediatrics Clinic, Emergency Clinical County Hospital, Tg. Mures, Romania
}

\begin{abstract}
Celiac disease $(C D)$ is an immune mediated disorder triggered by gluten diet genetically susceptible individuals. Celiac crisis is a rare but potentially lethal condition that can occur in a previously known celiac disease or as its first manifestation.

Case presentation. 15-year-old teenager, with a personal history of anemia for which he did not receive proper treatment, was admitted for semi-loose and steatorrheic stools, weight decrease, loss of appetite, fatigability, drowsiness, from approximately 10 days. The laboratory tests performed at the time of admission showed the following: thrombocytosis, iron deficiency anemia, slight increase of transaminases, mixed hyperbilirubinemia with the predominance of indirect bilirubin, and hyponatremia. We ruled out a potential infectious etiology, and the serology markers for celiac disease were positive. Thus, the diagnosis of celiac crisis has been established as the onset form of celiac disease based on the symptoms, increased anti-transglutaminase (>10x normal value) and anti-endomysium antibodies. During admission, we administered symptomatic treatment, fluids by vein, and we initiated the gluten-free diet. Three months after the initiation of the gluten-free diet, the patient's clinical course was favorable and the laboratory tests improved considerably: with negative anti-endomysium antibodies and marked decrease in anti-transglutaminase antibodies value.

Conclusions. The celiac crisis should be taken into account in the differential diagnosis of all patients with sudden onset diarrhea, metabolic disorders and weight loss after an infectious etiology has been ruled out, and its recognition is important and facilitates prompt treatment.
\end{abstract}

Keywords: celiac disease, celiac crisis, anti-transglutaminase antibodies, anti-endomysium antibodies, teenager

\begin{abstract}
Abbreviations
CD - celiac disease

GFD - gluten free diet

HLA - human leucocyte antigen

HGB - hemoglobin

HCT - hematocrit

VEM - mean erythrocyte volume

ALT - alanine aminotransferase

AST - aspartate aminotransferase
\end{abstract}
BiT - total bilirubin
BID - direct bilirubin
$\mathrm{Na}$ - sodium
ESR - erythrocyte sedimentation rate
$\mathrm{PCR}-\mathrm{C}$ reactive protein
IRM - magnetic resonance imaging
anti-TTG - tissue transglutaminase antibodies
EMA - anti-endomysium antibodies

\section{INTRODUCTION}

Celiac disease (CD) is an immune mediated disorder triggered by gluten diet genetically susceptible individuals. It is characterized by the presence of a variable combination of clinical manifestations, specific antibodies, enteropathy and HLA-DQ2 or
HLA-DQ8 haplotype [1]. CD has a multifactorial etiology, involving the interaction of genetic factors, gluten and environmental factors [2]. The overall prevalence of celiac disease is between $0.7 \%$ and $1.4 \%$ depending on age, gender and geographical location. In Europe, the prevalence is $0.8 \%$ [3]. Regarding the prevalence of CD in Romania, the 
available data is limited, with a $2.22 \%$ prevalence reported in the adult population in 2003 [4].

$\mathrm{CD}$ is frequently underdiagnosed due to the variability of clinical signs and symptoms. Typical symptoms include diarrhea, abdominal distension and pain, weight loss, malnutrition [5]. Extra-intestinal symptoms occur as a result of chronic malabsorption of micronutrients. Iron deficiency anemia is one of the main extra-intestinal symptoms, it often precedes diagnosis and, in many cases, it is the one that leads to further investigations for celiac disease. Other extra-intestinal manifestations are herpetiform dermatitis, changes in bone density, infertility, neurological manifestations, intestinal invagination [6], arthritis/ arthralgia, recurrent aphthous stomatitis, dental impairment, elevated transaminases [7]. CD can be asymptomatic, in which case, patients can be diagnosed by screening, indicated only in the increased risk groups [8].

Another form of presentation of celiac disease is the celiac crisis, a rare but potentially lethal disease. Clinically this is characterized by abdominal distension, severe diarrhea, vomiting that will lead to dehydration and hemodynamic instability, hypoproteinemia, electrolytic and metabolic disorders such as hypokalemia, hyponatremia, hypocalcemia, hypomagnesaemia [9]. Due to the acute onset and rapid progress of gastro-intestinal symptoms, patients usually require hospitalization. Recognizing this condition can be clinically difficult. The diagnosis of celiac crisis is based on acute symptoms, positive serology for celiac disease and histopathological examination. The treatment consists of hydro-electrolyte and nutritional support in the acute phase, corticosteroids in severe forms, and long-term GFD [10].

We present this case in order to highlight the atypical onset of CD in a teenager and the difficulties related to the diagnosis in case of celiac crisis.

\section{CASE PRESENTATION}

\section{The reasons for admission}

We report the case of a 15 year-old teenager, with a personal history of anemia for which he did not receive proper treatment, who was admitted for semiloose and steatorrheic stools, (2-3 stools/day), weight loss, approximately ( $8 \mathrm{~kg}$ in 10 days), inapetence, fatigability, drowsiness, with an onset of approximately 10 days.

\section{Clinical aspects}

The clinical exam at the time of admission highlighted circled eyes, pale skin, reduced skin turgor, poorly represented adipose tissue, severe abdominal distension and diffuse abdominal pain. The weight was $49 \mathrm{~kg}, 170 \mathrm{~cm}$ height, with a body mass index of $17 \mathrm{~kg} / \mathrm{m}^{2}$.

\section{Diagnostic criteria}

The laboratory tests at the time of admission revealed the following pathological elements: thrombocytosis $(605,000 / \mu \mathrm{l})$, moderate anemia $(\mathrm{Hgb} 9.4 \mathrm{~g} / \mathrm{dl}$, Hct $33.8 \%$, VEM $57 \mathrm{fl}$, moderate hypochromia with rare red blood cells in 'target' shape), low values of iron $(3.69 \mu \mathrm{mol} / \mathrm{l})$ and ferritin $(5.7 \mathrm{ng} / \mathrm{ml})$, mildly elevated transaminases (ALT 35.1 U/1, AST 39.8 U/1), mixed hyperbilirubinemia with the predominance of indirect bilirubin (BiT $3.03 \mathrm{mg}, \mathrm{BiD} 0.84 \mathrm{mg} / \mathrm{dl}$ ) and mild hyponatremia ( $\mathrm{Na} 134 \mathrm{mmol} / \mathrm{l})$. The examination of the stool for viral antigens and stool culture were negative, as well as the serology for viral hepatitis. Inflammatory markers (ESR, CRP) and serum albumin were within normal limits. The serology for celiac disease was positive: Anti-TTG (616.8 U/1) and EMA. Abdominal ultrasound revealed distended bowel loops, with fluid content, hyperechoic intestinal walls and accelerated bowel movements. The abdominal IRM revealed a significant distention of bowel loops, with fluid and gas accumulation, without wall thickening, except for an area on the ileum, and mesenteric reactive lymph nodes. Thus, diagnosis of celiac crisis was established as the onset form of celiac disease based on symptomatology, increased anti-TTG ( $>10 \mathrm{x}$ normal value) and positive EMA.

\section{Treatment and follow-up}

We administered fluids by vein, symptomatic treatment (Smecta, Metoclopramide, Hidrasec, Espumisan), probiotics and recommended the initiation of the GFD, with improvement of clinical and paraclinical parameters. The discharge recommendations were long-term GFD and symptomatic treatment in case of diarrhea or vomiting. After 3 months of GFD, the patient's clinical course was favorable (weight $63 \mathrm{~kg}$ ) and the laboratory parameters improved considerably: HGB $11.1 \mathrm{~g} / \mathrm{dl}$, VEM $66.1 \mathrm{fl}$, Hct 35.9\%, AST (22 U/l), ALT (16.3 U/1), BiT $(0.98 \mathrm{mg} / \mathrm{dl}), \operatorname{BiD}(0.34 \mathrm{ml} / \mathrm{dl})$, negative EMA and anti-TTG decreased to the value of $28.8 \mathrm{U} / \mathrm{ml}$.

\section{DISCUSSIONS}

$\mathrm{CD}$ is an immune mediated disease characterized by symptoms of malabsorption and gluten-triggered villous atrophy in genetically susceptible individuals. It was initially considered to be a rare condition of childhood, but it was proven to affect all age groups [11]. It is characterized by the presence of specific 
auto-antibodies directed against tissue transglutaminase, gliadin deamidate, endomysium, along with positive HLA-DQ2, HLA-DQ8 and intestinal histopathological changes [7]. Similarly, the age of the patient described above was 15 years. CD in children often manifests itself with gastrointestinal symptoms (chronic diarrhea, vomiting, appetite loss, abdominal distension, abdominal pain, failure to thrive). They may also show extra-intestinal symptoms or may even be asymptomatic. Sometimes CD can have an acute onset in the form of celiac crisis. The celiac crisis usually has severe gastro-intestinal manifestations, hypoproteinemia, electrolyte and metabolic disorders that require hospitalization [12]. Celiac crisis is the term used in patients with acute or severe symptoms and potentially fatal consequences. This can be the first form of manifestation or it can be a complication in patients diagnosed with celiac disease [9]. Similarly, we described the case of an apparently healthy teenager, who presented diarrhea, vomiting, hyponatremia and weight lost, as the onset of celiac disease, which is part of the celiac crisis clinical spectrum. Jamma et al developed several criteria for the diagnosis of celiac crisis in adult patients [9]. This condition has been defined as: acute onset or rapid progression of gastrointestinal symptoms due to celiac disease requiring hospitalization and/or parenteral nutrition together with at least 2 of the following: signs of severe dehydration including hemodynamic instability; neurological impairment; renal dysfunction with a creatinine level above $2.0 \mathrm{~g} / \mathrm{dl}$; metabolic acidosis, $\mathrm{pH}$ less than 7.35; hypoproteinemia (albumin level below $3.0 \mathrm{~g} / \mathrm{dl}$ ); electrolytic anomalies (hypernatremia/hyponatremia, hypocalcemia, hypokalemia or hypomagnesaemia); weight loss, more than 4,5 $\mathrm{kg}$ [9]. Since data in the literature data are limited in terms of diagnostic criteria for pediatric celiac crisis, most information originating from studies performed on adults, the age groups of children and adolescents remain less studied. Thus, in the case of our patient the following criteria were met from those described above: the acute onset of

\section{REFERENCES}

1. Husby S, Koletzko S, Korponay-Szabó IR et al. European Society for Pediatric Gastroenterology, Hepatology, and Nutrition Guidelines for the Diagnosis of Coeliac Disease. J Pediatr Gastroenterol Nutr. 2012;54(1):136-60.

2. Sollid LM, Lie BA. Celiac disease genetics: current concepts and practical applications. Clin Gastroenterol Hepatol. 2005;3:843-51.

3. Singh P, Arora P, Strand TA et al. Global Prevalence of Celiac Disease: Systematic Review and Meta-analysis. Clin Gastroenterol Hepatol. 2018;16(6):823-836.e2.

4. Dobru D, Pascu O, Tanță M et al. The prevalence of coeliac disease at endoscopy units in Romania: routine biopsies during gastroscopy the intestinal symptoms requiring hospitalization, hyponatremia and weight loss. Other authors pointed out that this form of celiac disease is triggered by a specific factor, such as previous surgical intervention, infections, pregnancy [9], hypoproteinemia, lack of compliance to GFD, anticholinergic drugs [13]. These factors seem to trigger an activation of the immune system with severe inflammation of the bowel mucosa and a perturbation of the normal bowel motility [9]. In our case we found no history of surgical intervention or use of anticholinergic drugs, and infection was ruled during admission. The only plausible trigger factor could be the lack of a GFD since our patient presented no history of previously diagnosed CD.

Even though the association between Gilbert syndrome and $\mathrm{CD}$ has been previously described in the literature [14], the information is scarce. Thus, in our case, mixed hyperbilirubinemia mixed with the predominance of indirect bilirubin, prolonged anorexia and recent low-calorie intake, prior to the presentation, suggest the possible association of a Gilbert syndrome.

Therapeutic management in celiac crisis requires hydro-electrolyte rebalancing, nutritional support and the initiation of GFD. In severe cases, corticosteroids are also required [10]. In this case, fluid and electrolytic rebalancing as well as the initiation of GFD had a favorable outcome.

\section{CONCLUSIONS}

The diagnosis of celiac crisis represents a challenge, especially in patients who were not previously diagnosed with $\mathrm{CD}$. Therefore, this condition should always be taken into account in the differential diagnosis of all patients with diarrhea and suggen onset of metabolic disturbances associated with weight loss after the exclusions of an infectious pathology. In conclusion, we emphasize that recognizing the acute onset of celiac disease in the form of a celiac crisis is important and facilitates its prompt treatment.

\section{Conflict of interest: none declared Financial support: none declared}

are mandatory (a multicentre study). Rom J Gastroenterol. 2003; 12:97-100.

5. Glissen Brown JR, Singh P. Coeliac disease. Pediatr Int Child Health. 2019;39(1):23-31.

6. Al-Bawardy B, Chamil Codipilly D, Rubio-Tapia A et al. Celiac disease: a clinical review. Abdom Radiol. 2017;42(2):351-360.

7. Husby S, Koletzko S, Korponay-Szabo I et al. European Society Paediatric Gastroenterology Hepatology and Nutrition Guidelines for Diagnosing Coeliac Disease 2020. J Pediatr Gastroenterol Nutr. 2020;70:141-156.

8. Lindfors K, Ciacci C, Kurppa K et al. Coeliac disease. Nat Rev Dis Primers. 2019;5(1):3. 
9. Jamma S, Rubio-Tapia A, Kelly CP et al. Celiac crisis is a rare but serious complication of celiac disease in adults. Clin Gastroenterol Hepatol. 2010;8:587-590.

10. Balaban DV, Dima A, Jurcut $C$ et al. Celiac crisis, a rare occurrence in adult celiac disease: A systematic review. World J Clin Cases. 2019;7(3):311-319.

11. Green PHR, Cellier C. Celiac disease. N Engl J Med. 2007;357(17):1731-1743.

12. de Almeida Menezes M, Cabral V, Silva Lorena SL. Celiac crisis in adults: a case report and review of the literature focusing in the prevention of refeeding syndrome. Rev Esp Enferm Dig. 2017; 109(1):67-68.

13. Mones RL, Atienza KV, Youssef NN et el. Celiac Crisis in the Modern Era. J Pediatr Nutr. 2007;45(4):480-483.

14. Kocsis D, CsaplárM, Jócsák E et al. Celiac disease association with other autoimmune disorders: three case reports. Case Rep Int Med. 2015;2:23-9. 